\title{
The Position of Moving Objects
}

Eagleman and Sejnowski (1) showed that the flash-lag effect $(2,3)-a$ visual illusion in which two objects physically at the same location appear to be offset because of differing motion trajectories - cannot be explained by latency correction (4) or by differential latencies for flashed and moving objects (5-7). Our work, like theirs, has found that the flash-lag effect is due to the motion of the moving object after the flash, and we too have proposed that the perceived position of flashed and moving objects is based on temporal integration $(8-10)$. We disagree, however, with the statement by Eagleman and Sejnowski that the flash "resets" this integration, and we see no need to "postdict" the perceived offset to the time of stimulus presentation.

To test the temporal-integration hypothesis, we used a stimulus, originally designed by Baldo and Klein (11), that involved seven dots,
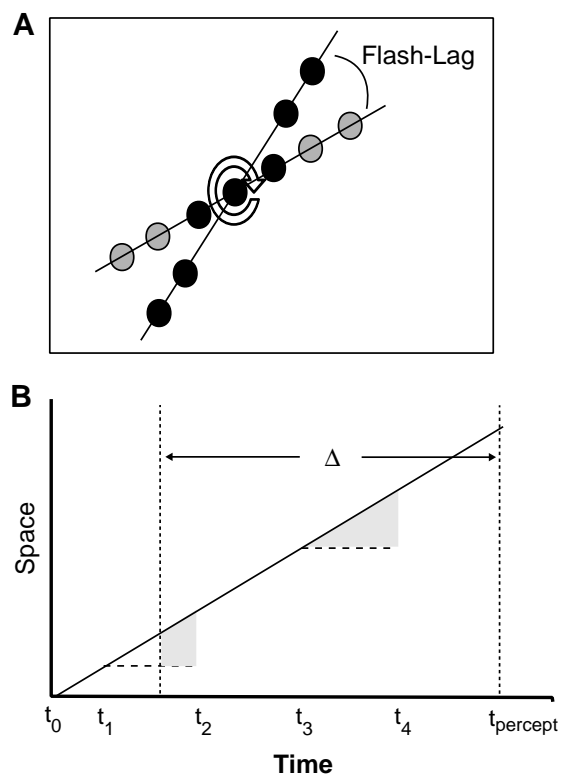

Fig. 1. The flash-lag effect and temporal integration of position signals. (A) The flash-lag stimulus. Seven dots in perfect alignment rotate around the central fixation point. The outer dots (gray) are visible only intermittently; the inner dots are continuously visible. Observers perceive a spatial offset between the outer, flashed dots and the inner dots (black dots show the percept). (B) The temporal-integration hypothesis as a space-time diagram. The motion trajectory of the inner dots is represented as the solid line; the outer dots are visible only from $t_{0}$ to $t_{1}$, from $t_{2}$ to $t_{3}$, and from $t_{4}$ onwards. When the outer dots are invisible, we assume that an internal position signal represents their last visible position (dashed line). The percept at time $t_{\text {percept }}$ is the average difference of the two position signals (solid and dashed lines) over a temporal-integration window $\Delta$. This average is equivalent to the shaded area divided by $\Delta$. all of which rotated around the central fixation point (Fig. 1A). The inner three dots were continuously visible; the outer dots could be made visible or invisible at various times. Turning the dots on for only one videoframe at the start of the rotational movement led to a large flash-lag effect. If the dots were switched on again in a later videoframe (again in perfect physical alignment with the inner dots), the flash-lag was much reduced. Thus, the temporal integration did not stop between flashes, nor did the second flash reset the integration. By varying the number of flashes and the time between them, we were able to show that the temporal integration for this particular stimulus configuration extends over approximately half a second $(8-10)$.

Figure 1B explains our temporal-integration hypothesis. The offset between two objects that is perceived at time $t$ is given by the average of the difference of the two position signals over a time window $\Delta$ that precedes the moment of perception. In that time window, the moving object occupies multiple visible positions, but the flashed object is hidden. We suggest that the integration mechanism will, in the absence of an updated position signal, resort to using the last visible position of the flashed object. A temporal integration on the order of half a second quantitatively agrees with the data. Moreover, the model predicts that as more of the motion trajectory of the flashed object becomes visible, less flash-lag should remain, and that is indeed the case $(8,9,12)$. Another prediction of the model is that it should be possible to abolish the flash-lag effect by halting the moving object after the flash or between multiple flashes. We confirmed that prediction for multiple flashes (10), and Eagleman and Sejnowski showed it to hold for single flashes [figure 1 of (1)].

Our work suggests that there is no need to postdict the perceived offset to the time of the flash. The causal chain of events is unambiguous: light hits the retina, neural mechanisms start processing the stimulus, and after some time a decision is reached to answer the question the experimenter asked. The observer need not relate this decision time to the time at which the light from the stimulus hit the retina, which only the experimenter knows. That motion after the flash influences the percept generated by that flash merely shows that visual processing takes time. That should be uncontroversial.

\section{Bart Krekelberg Markus Lappe \\ Department of Neurobiology Ruhr University Bochum Bochum 44780, Germany E-mail: bart@neurobiologie. ruhr-uni-bochum.de}

References

1. D. M. Eagleman and T. J. Sejnowski, Science 287, 2036 (2000).

2. W. Metzger, Psychologische Forschung 16, 176 (1931).

3. D. M. Mackay, Nature 181, 507 (1958).

4. R. Nijhawan, Nature 370, 256 (1994).

5. G. Purushothaman, S. S Patel, H. E. Bedell, H. Ögmen, Nature 396, 424 (1998)

6. D. Whitney and I. Murakami, Nature Neurosci. 1, 656 (1998).

7. , P. Cavanagh, Vision Res. 40, 137 (2000).

8. M. Lappe and B. Krekelberg, Perception 27, 1437 (1998).

9. B. Krekelberg and M. Lappe, Vision Res. 39, 2669 (1999).

10.

11. M. V. Baldo and S. A. Klein, Nature 378, 565 (1995).

12. T. Bachmann and K. Kalev, Perception S26, 119 (1997).

10 April 2000; accepted 21 July 2000

Eagleman and Sejnowski (1) recently proposed a "postdiction" model of the so-called flash-lag effect, in which a moving stimulus appears spatially to lead a flash, even though both stimuli are actually precisely aligned (2). According to postdiction, the moving object appears ahead of the flash because at each moment the object's position is estimated by integrating forward in time; the flash resets all the integrals so that only those starting immediately after the flash will produce a position estimate, and the forward average is necessarily in advance of the position of the flash.

Eagleman and Sejnowski suggested that this flash-reset mechanism also explains why a moving object that abruptly appears and starts to move is initially invisible for some distance, a phenomenon known as the Fröhlich effect (3). In the postdiction model, the abrupt onset of a moving object acts as a flash, so the first perceived position of the object, integrated during the following $\sim 80$ ms, is again well ahead of the object's actual first position. Alternative explanations for the flash-lag effect (4-7), by contrast, including our model of differential latencies for flashed and moving objects (5), are unable to account for the Fröhlich effect. Eagleman and Sejnowski conclude that if the flash-lag effect and the Fröhlich effect are caused by the same mechanism, and our differential-latency model fails to explain the Fröhlich effect, then our model must also be rejected as an explanation of the flash-lag phenomenon (1).

A closer examination, however, shows that postdiction explains neither the flash-lag effect nor the Fröhlich effect, and that our differential-latency model remains a viable account of the flash-lag phenomenon. According to postdiction, the flash resets all the ongoing motion integrals. That should render any nearby moving object invisible for the 80 ms before the flash's appearance, as occurs at the onset of motion in the Fröhlich effect. Thus, a series of flashes, each of them aligned with a moving object (within $<80 \mathrm{~ms}$ of each 


\section{TECHNICALCOMMENTS}

other), should continually reset all the integrals before any value is determined, completely masking the moving stimulus. When a series of aligned flashes is presented sequentially (7), however, the visibility of the motion is unaffected, and an undiminished flashlag effect is seen for each individual flash.

In addition, contrary to the hypothesis of Eagleman and Sejnowski, we find that a single mechanism cannot account for both the flash-lag and Fröhlich effects (Fig. 1). When a stationary object was presented for $2500 \mathrm{~ms}$ ("stationary cue" in Fig. 1A), removed for 30 $\mathrm{ms}$, and then presented again in immediate motion, the extent of the initial trajectory over which the object remained invisible was greatly reduced or eliminated (blue symbols in Fig. 1B). According to postdiction, the invisibility of motion initiation should not depend on events before the onset of motion, because the motion onset itself resets all ongoing integrals. Our data show that this is not

A
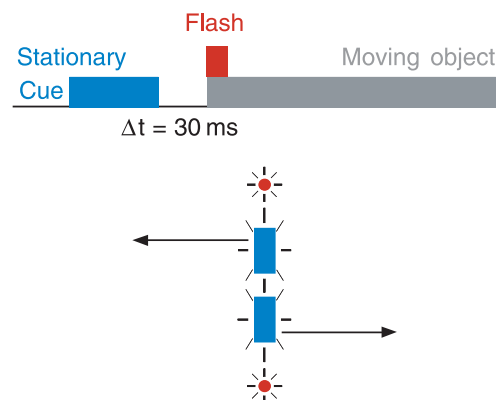

B

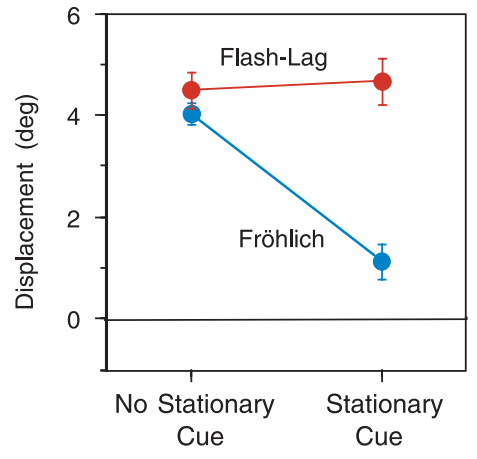

Fig. 1. Stimulus time course and data distinguishing the flash-lag effect from the Fröhlich effect. (A) Two stationary objects were presented for $2.5 \mathrm{sec}$ (stationary cue), then removed for $30 \mathrm{~ms}$. After that $\Delta t$, the objects were presented again and immediately began to move in opposite directions (to avoid eye movements). In a second experimental condition, no stationary cue was presented. (B) Blue symbols refer to the perceived Fröhlich effect, the degree to which the initial position of the moving objects appeared shifted in the direction of their motion. Red symbols refer to the perceived flash-lag effect, the degree to which the flashes appeared to trail behind the position of the moving objects. The flash-lag effect was constant whether the cue was presented or not; the Fröhlich effect was reduced dramatically when the cue was presented. the case. The cue overrode the initial invisibility of the moving object and, thus, effectively abolished the Fröhlich effect; yet, regardless of whether the cue was presented, an adjacent flash $(15 \mathrm{~ms})$ aligned with the motion initiation appeared to lag behind the moving object, and the flash-lag effect remained (red symbols in Fig. 1B).

Our results show that the Fröhlich effect - the delayed onset of visibility of a target set abruptly in motion-is not a flash-lag effect. When Eagleman and Sejnowski claimed, in their third experiment, to have disproved the differential-latency model, they based their claim on the onset of visibility, that is, on the Fröhlich effect. That is not a test of our model. Our results with the stationary cue are consistent with previous suggestions that the Fröhlich effect involves attention and possibly metacontrast masking $(8)$.

Postdiction thus is unable to account for the flash-lag and Fröhlich effects, because the two phenomena are actually caused by distinct mechanisms. Our differential latency model (5) remains a viable explanation of flash-lag data.

\section{David Whitney Patrick Cavanagh Vision Sciences Laboratory Harvard University 33 Kirkland Street Cambridge, MA 02138, USA E-mail:whitney@wjh.harvard.edu}

References

1. D. M. Eagleman and T. J. Sejnowski, Science 287, 2036 (2000).

2. R. Nijhawan, Nature 370, 256 (1994).

3. F. W. Fröhlich, Die Empfindungszeit (Verlag von Gustav Fischer, Jena, Germany, 1929).

4. M. V. Baldo and S. A. Klein, Nature 378, 565 (1995).

5. D. Whitney and I. Murakami, Nature Neurosci. 1, 656 (1998).

6. G. Purushothaman, S. S. Patel, H. E. Bedell, H. Ögmen, Nature 396, 424 (1998).

7. M. Lappe and B. Krekelberg, Perception 27, 1437 (1998).

8. K. Kirschfeld and T. Kammer, Vision Res. 39, 3702 (1999).

17 May 2000; accepted 21 July 2000

Response: Both the comment of Krekelberg and Lappe and that of Whitney and Cavanagh question our interpretation of the flash-lag effect and propose different explanatory models. However, both their results $(1-5)$ and ours $(6)$ can be explained within the postdictive framework we have proposed.

Specifically, the authors of both comments disagree with our hypothesis that the flash resets motion integration (i.e., previously collected information). We assume that the visual system has an internal model of external visual stimuli and that this internal model is compared continuously with new external measurements (7). If an unpredicted transient (such as a flash) occurs, it may be advantageous to temporarily devalue the internal model and to rely more heavily on subsequent measurements. This need not be an all-or-none switch, however, as the authors of the comments apparently assume. Instead, the amount of information discarded will likely be graded and will depend on the salience of the transient stimulus: The greater the surprise, the less the internal model is relied upon. We hypothesize further that visual awareness requires a match between the internal model and external measurements, which is disrupted by the transient stimulus.

In the flash-lag effect, the internal model of the moving object is strongly discounted (i.e., reset) by the flash, and a new internal model of the moving stimulus is generated de novo; this situation yields the Fröhlich effect. Whitney and Cavanagh argue that the flashlag effect is not an expression of the Fröhlich effect. In the experiments that they report to establish that argument, however, more than one parameter was changed between conditions. In one condition (stationary cue, no flash), the brief offset of the cue was only moderately salient, and thus the illusory displacement that followed was small. The other three conditions, by contrast, contained a flash, or the sudden, unpredicted onset of the square, or both; under those conditions, the salience of the stimulus was higher and the illusory displacement was larger, as we would predict. The experiments of Whitney and Cavanagh thus do not rule out the relationship between the flash-lag effect and the Fröhlich effect, but rather serve to illustrate

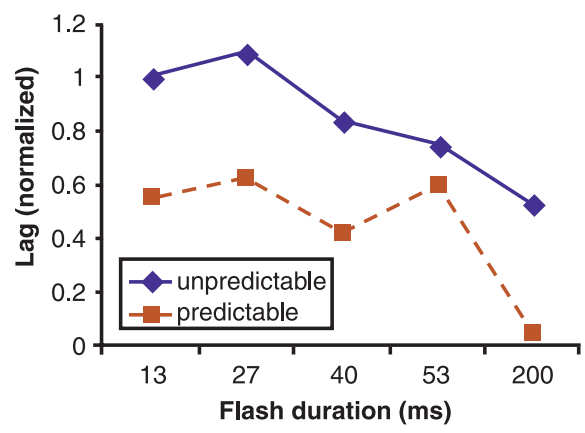

Fig. 1. Role of predictability in the flash-lag effect. Experimental conditions replicate those of (5): three central dots spin at $0.5 \mathrm{~Hz}$; two pairs of flanking dots are flashed with an offset angle. Illusory displacement is quantified by a method of adjustment at five flash durations (six trials each duration). Each trial reflects of one of two randomly interleaved conditions: the flashes appear once every $2000 \mathrm{msec}$ at the same location each time (predictable, squares), or they appear randomly from 1500 to 2500 ms after the last flash and, thus, at random locations (unpredictable, diamonds). Data on each subject are normalized to the magnitude of the lag angle in the unpredictable condition at $13 \mathrm{~ms}$ (absolute lag angles for that condition, $n=6$ subjects: $9.2^{\circ}, 10^{\circ}, 6.7^{\circ}, 8.3^{\circ}, 19.2^{\circ}$, and $\left.17.5^{\circ}\right)$. Demonstrations of stimuli can be found at www.cnl.salk.edu/ eagleman/flashlag. 


\section{TECHNICALCOMMENTS}

our hypothesis that differentially salient stimuli will engender differential amounts of perceived displacement.

As a further test of our hypothesis, we replicated the multiple-flash experiment of Krekelberg and Lappe $(3,5)$, but with a new condition: Instead of the outer dots flashing periodically and predictably, on half the trials the outer dots were flashed at unpredictable times but at the same average rate. The degree of perceived displacement in the unpredictable case was approximately twice as large (Fig. 1). Our interpretation is that the internal model is less able to predict the next appearance of the flash for the aperiodic stimuli, and as a consequence suffers more devaluation due to surprise. The Krekelberg and Lappe model does not predict these results because it does not take into account the salience of a signal, but only its positional difference within a fixed temporal window.
A detailed discussion of the possible neural mechanisms underlying the flash-lag effect is beyond the scope of this discussion. Ultimately, however, the difference between our explanation and those of the comment authors is that, whereas they propose purely "feed-forward" models, our framework requires feedback, an important architectural feature of the cerebral cortex. The term "postdiction" captures this simple but crucial idea. Additional support for postdiction is seen in well-established phenomena such as backward masking and apparent motion, and our hypothesis has found support in recent experiments $(8,9)$.

David M. Eagleman

Sloan Center for Theoretical Neurobiology The Salk Institute for Biological Studies 10010 North Torrey Pines Road La Jolla, CA 92037, USA E-mail: eagleman@salk.edu
Terrence J. Sejnowski

Howard Hughes Medical Institute

The Salk Institute for Biological Studies and Department of Biology

University of California at San Diego La Jolla, CA 92093, USA

References

1. D. Whitney and I. Murakami, Nature Neurosci. 1, 656 (1998).

2. , P. Cavanagh, Vision Res. 40, 137 (2000).

3. B. Krekelberg and M. Lappe, Vision Res. 39, 2669 (1999).

4. , Vision Res. 40, 201 (2000)

5. M. Lappe and B. Krekelberg, Perception 27, 1437 (1998).

6. D. M. Eagleman and T. J. Sejnowski, Science 287 2036 (2000).

7. R. P. Rao, Vision Res. 39, 1963 (1999).

8. B. R. Sheth, R. Nijhawan, S. Shimojo, Nature Neurosci. 3, 489 (2000)

9. E. Brenner and J. B. Smeets, Vision Res. 40, 1645 (2000).

26 June 2000; accepted 21 July 2000 